\title{
Homeostatic generative design process: Emergence of the adaptive architectural form and skin to excessive solar radiation
}

International Journal of

Architectural Computing

$1-16$

(C) The Author(s) 2020

Article reuse guidelines: sagepub.com/journals-permissions DOI: I0.1 I77//478077/2095/947 journals.sagepub.com/home/jac

@SAGE

\author{
Milad Showkatbakhsh ${ }^{\prime}\left(\right.$ iD and Saam Kaviani ${ }^{2}$
}

\begin{abstract}
Natural organisms through their evolutionary developments, acquire adaptive morphological and behavioural characteristics within their environmental contexts. Through homeostatic behaviours, organisms, individually and collectively, will sustain internal and external equilibrium in face of environmental fluctuations. There is a wide range of morphological and behavioural traits across multiple species that are rooted in their homeostatic mechanisms throughout their lives. This paper presents an evolutionary design workflow with embedded homeostatic principles to generate a building cluster that is adapted to the contexts with extreme solar radiation.
\end{abstract}

\section{Keywords}

Architecture, computation, evolution, biology, homeostasis, morphology, skin, genetic algorithm, Computer-Aided Design

\section{Introduction}

Achieving thermal comfort in the architectural spaces that are located in regions with excessive solar radiations has always been a challenging task. The European Council reports that buildings currently consume an estimated $40 \%$ of the region's energy (predominantly for cooling and heating mechanisms) and are responsible for up to $36 \%$ of the European Union's CO2 emissions. ${ }^{1}$

Natural systems are the source of inspiration for solving complex real-world problems across different disciplines. Evolution is the mechanism by which natural systems have evolved and is compelling to investigate to derive a design methodology for problems of adaptation to the environment. Through understanding the significance of the homeostatic processes of species in their evolutionary developments, a generative design process is proposed in this paper that stresses the significance of the morphological properties of buildings in their adaptation to excessive solar radiation.

\footnotetext{
'School of Architecture, Architectural Association, London, UK

${ }^{2}$ School of the Built Environment and Architecture, London South Bank University, London, UK

Corresponding author:

Milad Showkatbakhsh, School of Architecture, Architectural Association, 36 Bedford Square, Fitzrovia, London WCIB 3ES, UK. Emails: showkatbakhsh@aaschool.ac.uk; milad.showkatbakhsh@gmail.com
} 
The existing thermal regulatory systems have contributed to the loss of a significant amount of energy through implemented heating and cooling mechanisms. ${ }^{2}$ The cooling systems utilise more energy in summer than the heating systems in winter. These systems are being utilised extensively in the countries with excessive solar radiation; for example, the Persian Gulf region, and result in extreme electricity loads. ${ }^{3}$ The European Union funding numerous projects to advance the research in this sector ${ }^{4}$ is indicative of the urgency of this problem. There is an escalating need to consider these factors, not only in the later stages of design (i.e. insulation) but in the early stages of design development. Is it possible to utilise principles of homeostasis and evolution in nature in the design processes to effectively address a set of conflicting objectives at the early stages of design explorations? This research addresses this question by demonstrating a novel generative design system with embedded homeostatic characteristics to generate a family of solutions that address a set of conflicting objectives.

\section{Natural systems: homeostasis and morphology}

Species have evolved morphological characteristics, responsive behaviours and processes that help to sustain the exchange of matter and energy with their environment. These exchanges are monitored and maintained through responsive regulatory mechanisms. Homeostasis (the term coined initially by $\mathrm{Cannon}^{5}$ ) is a responsive process by which the organism can sustain a steady-state through internal and external changes. This process is comprised of sensing, decision-making and reactions that are widely distributed in the skin and the internal organs.

The 'self-regulatory' aspect of homeostatic processes, according to Gerlee et al. will affect the final form and function of a system. ${ }^{6}$ This process occurs across the different levels of organisms and controls a wide range of parameters, one of which is heat. The homeostatic process that controls heat is called thermoregulation.

The objective of thermoregulation is to cancel the effects of an unfavourable change in the temperature of the system. This process comprises three main elements: (1) A setpoint, (2) Receptors (3) Effectors. ${ }^{7}$ Morphological traits are indispensable for successful thermoregulation of species and are manifested across a range of scales, one of which is skin. The biological and physical characteristics of the skin play an enormous role in protecting the body from the environmental stimulus.

\section{Relevance in design}

As noted in the differences between the revised and the original editions of Steadman, 1979, 'The Evolution of Designs', principles of natural systems and processes can be implemented in the design disciplines to drive the outcome in closer conformity to its environment. ${ }^{8}$

Evolutionary design processes have been used as robust problem-solving strategies in architecture and design disciplines since the 1950s. Given the importance of the thermoregulation of species in their evolutionary development, ${ }^{9}$ the application of these regulatory methods as feedback systems in the generative design process is investigated in this paper.

\section{Literature review: computational platform}

Evolutionary multi-objective optimisation strategies have been utilised widely since the late 20th century as problem-solving methods. Sewell Wright in the 1930s is the earliest instance of the application of evolutionary principles as optimisation processes. ${ }^{10}$

Ernst Mayr described the evolutionary model as a two-step process; random variation within the genome (instructions) of a phenotype (geometry), and subsequently the selection of the phenotype through environmental pressures. ${ }^{11}$ Homeostatic processes such as thermoregulation are iterative in principle. Thus their 


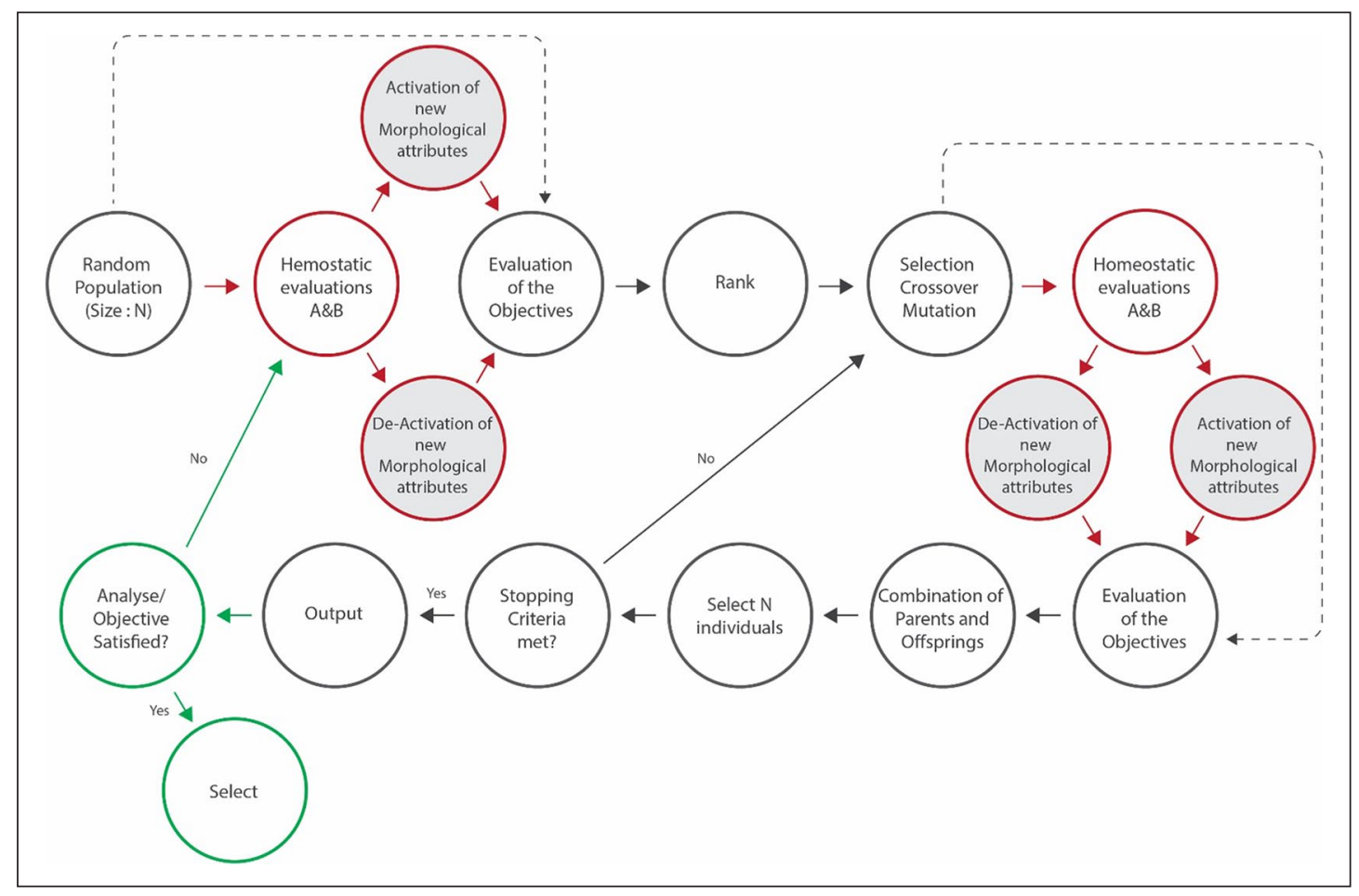

Figure I. The modified evolutionary simulation workflow with two secondary evaluation mechanisms. The red circles and arrows show the modified stages. The green circles and arrows show the added step to enhance application of evolutionary simulation in design using Wallacei. The grey circles and arrows show the NSGA II algorithm pseudocode.

application in the design processes requires an iterative generative model that includes a mechanism of assessment and reconfiguration of the generated results. In this context, heat received by solar radiation is the parameter for which the homeostatic mechanism will be investigated by inserting the secondary evaluation mechanisms into the evolutionary simulation (Figure 1). Thus, the experiment presented in this paper investigates to what extent the application of thermoregulatory principles as feedback mechanisms in the evolutionary simulations will be efficient to generate adaptive forms.

The main components of a homeostatic process (setpoint, receptor and effector) are mapped to a set of algorithmic conditional statements within the main generative design problem algorithm.

The presented experiment utilises multi-objective Non-Dominated Sorting Genetic Algorithm II (NSGAII) developed by Deb et al. ${ }^{12}$ as the base algorithm based on which the generative simulation is developed. Rhinoceros3D, Grasshopper3D and its plugin 'Wallacei'13 are used to run the simulation and analyse the results thoroughly. In the conducted experiments, the algorithm parameters within the evolutionary simulation were set to the following values (Table 1) (for a detailed description of the terminology used in the simulation, please see Makki et al. $\left.{ }^{14,15}\right)$.

\section{Case study}

The Persian Gulf region has extreme sun radiations in summer. The temperature of this region can rise to $60^{\circ} \mathrm{C}$ in summers. ${ }^{16}$ The $\mathrm{Al} \mathrm{Bahr}$ project, which is located in Abu Dhabi's financial centre, comprises two identical $145 \mathrm{~m}$ tall towers with 26 stories each (Figure $2^{17,18}$ ). 
Table I. Simulation parameters.

\begin{tabular}{llc}
\hline Parameter & Short description & Value \\
\hline Generation size & Number of individuals per generation & 25 \\
Generation count & Number of generations in the simulation & 300 \\
Crossover probability & Percentage of solutions that reproduce in each generation & 0.9 \\
Mutation probability & The percentage of mutation I/ (number of variables) & $1 / \mathrm{n}$ \\
Crossover distribution index & Probability of similarity of the offspring to the parents & 20 \\
Mutation distribution index & Probability of similarity of the offspring to the parents & 20 \\
Random seed & Random seed in the simulation & I \\
\hline
\end{tabular}

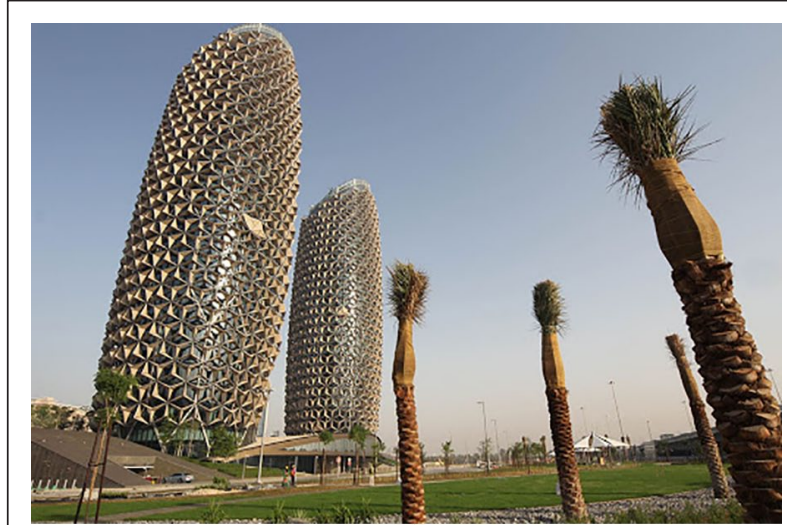

(a)

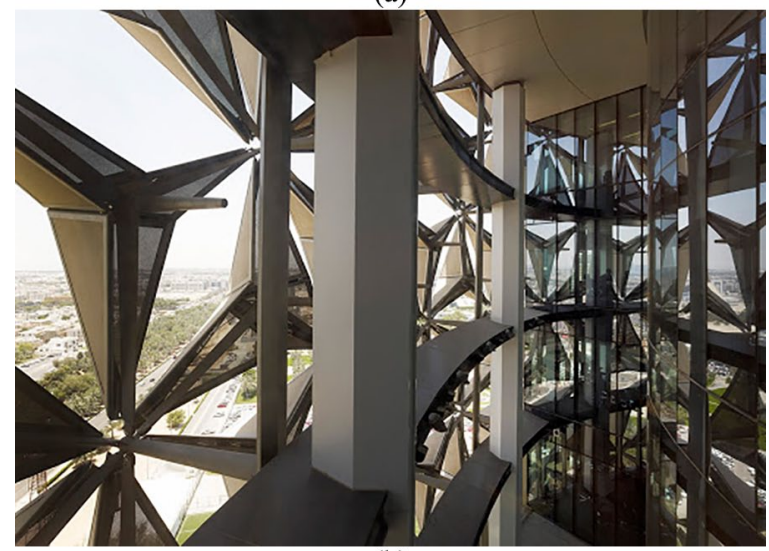

(b)

Figure 2. (a) Al-Bahr towers. (b) The view from inside to the outside of Al-Bahr tower.

The implemented skin system in the Al-Bahr project is the reason for choosing this project as the case study. Based on the results of the surveys on the evaluation of adaptive facades, ${ }^{3} 60 \%$ of the users are uncomfortable with the natural light that is received through the skin. Given the uniqueness of the project skin system, the overall morphology of the towers comprises no formal attributes suitable for the exposed excessive solar radiation. The complexity of the skin and the simplicity of the form of the Towers were the reason to investigate the skin to morphology relationship in this paper. 


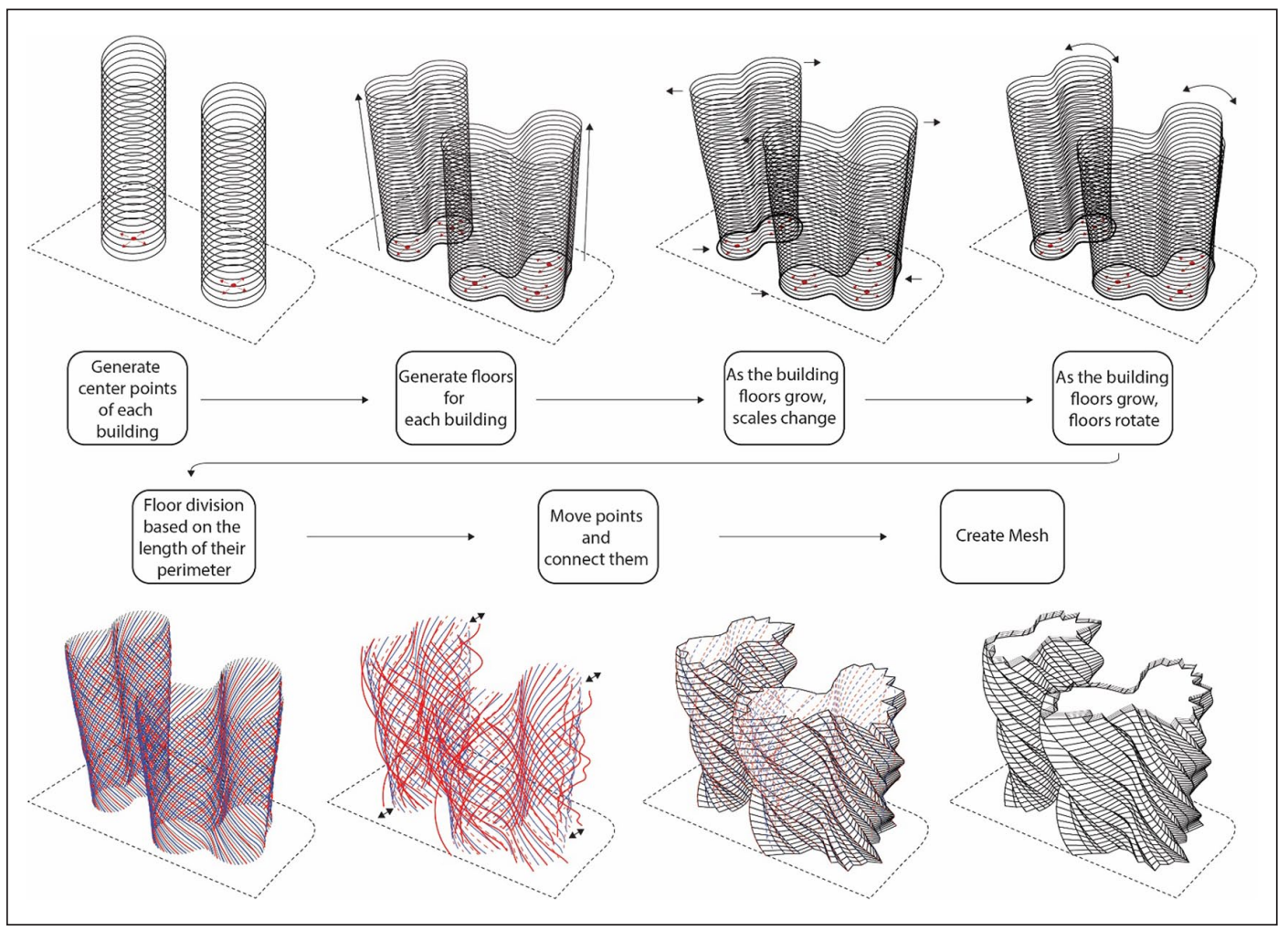

Figure 3. The algorithmic construction of the primitive geometry based on Al-Bahr towers geometry.

\section{Experiment setup}

In the context of this paper, the summer solstice (21st June) is considered the date on which solar radiation is calculated and studied, and it is the only way by which heat can be exchanged. Therefore, the primary aspect of the experiment is examining the significance of the form and skin in adaptation to excessive solar radiation. The form and skin will be generated through generative simulation with homeostatic feedback mechanisms. The selected group of generated solutions will be compared to the Al-Bahr Towers.

The form of Al-Bahr Towers was used to construct a primitive geometry algorithmically with added modifications to enable the changes necessary in the simulation (Figure 3).

\section{Homeostatic feedback mechanism}

Two secondary feedback mechanisms are added to the generative simulation to drive it towards generating an extra set of formal attributes within the geometry. They are called homeostatic mechanism A and homeostatic mechanism B.

Homeostatic Mechanism A (H-M-A) directs the simulation to generate the skin system on the buildings should a specific condition be met. Its three main elements can be identified as follows: (Figure 4) (Table 2).

- Setpoint: $60 \%$ of solar radiation exposure on the building's form is the threshold after which a responsive feedback mechanism will be activated in order to counteract the deviation (the authors chose the figure $60 \%$ as a design input; this number can be modified accordingly in other scenarios). 


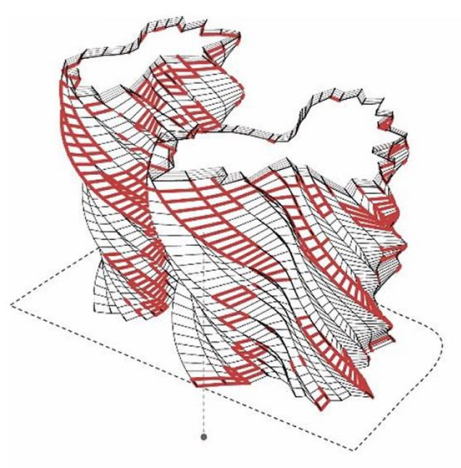

Parts of the form where solar radiation is more than $60 \%$

$F(x)$

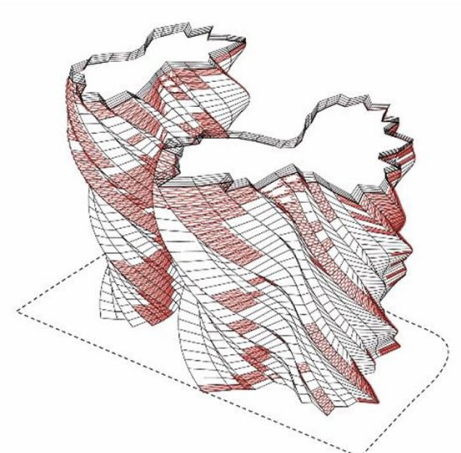

- Activation of a new formal attribute, a Skin system

$\mathrm{G}(\mathrm{x})$

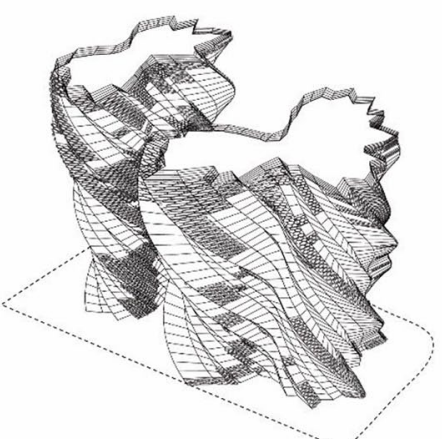

(a)
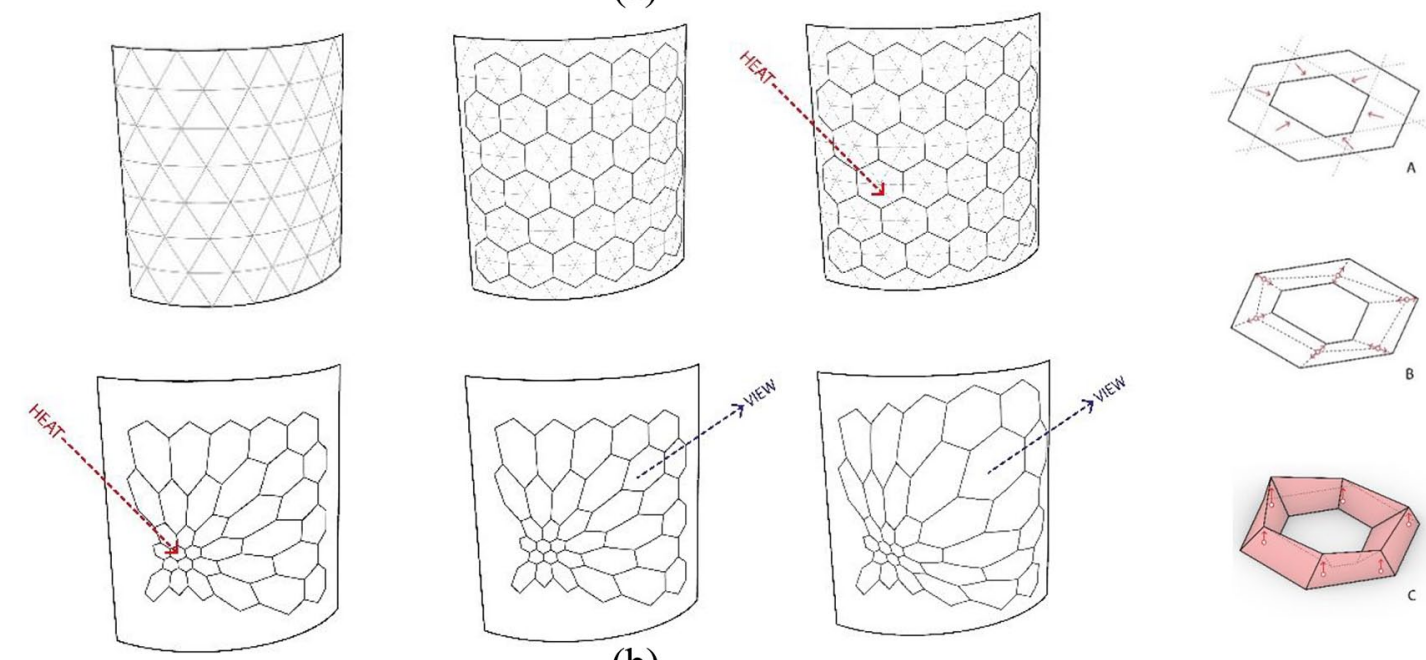

(b)

Figure 4. Illustration of (a) H-M-A process and effects (skin system); (b) algorithmic setup of the skin system.

- Receptor: Building forms have been created algorithmically based on Figure 3, allowing for the evaluation of the solar radiation on the building surfaces.

- Effector: If the amount of solar radiation exceeds $60 \%$, a new morphological attribute, skin, will be activated on the exposed surfaces.

$$
\begin{gathered}
\operatorname{if}(\mathrm{F}(\mathrm{X}) !=\text { null }) \\
\mathrm{G}(\mathrm{X})
\end{gathered}
$$

else

X; 
Table 2. Homeostatic mechanism A functions.

\begin{tabular}{ll}
\hline Functions and variables & Description \\
\hline $\mathrm{X}$ & The generated phenotype in each iteration \\
$\mathrm{F}()$ & $\begin{array}{l}\text { Receptor. The function that returns the parts of the phenotype that receive solar } \\
\text { radiation more than } 60 \%\end{array}$ \\
$\mathrm{G}()$ & Effector. The Function that applies the morphological changes onto the phenotype
\end{tabular}

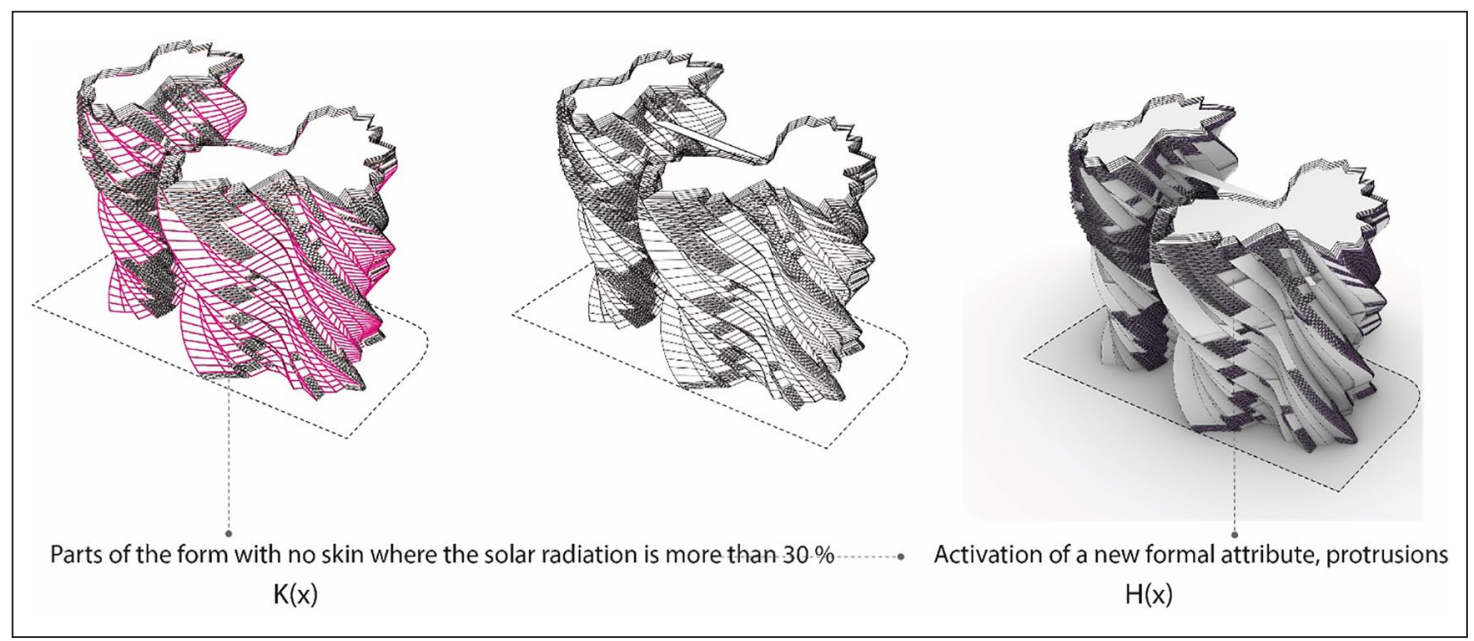

Figure 5. Illustration of H-M-B process and effects (protrusions).

The effectiveness of the skin in terms of blocking the excessive solar radiations as well as providing a good view to outside relies on the elements that are considered as the variable values of the shapes of building skin such as the number of modules, offset size, extrusion size.

By distinguishing the values of the building skin that are fixed and variable, during the early design exploration stage, geometrical adaptability of the skin can be tested to ensure the emergent skin, that may evolve throughout the simulation can be applied to any complex form. The process of setting up the variables and development is illustrated in Figure 4. The hexagons are chosen as the skin system in this experiment as an example to show that even complex geometries can take advantage of homeostatic principles.

Homeostatic Mechanism B (H-M-B) affects areas of the buildings with no skin system. Its three main elements are as follows (Figure 5) (Table 3):

- Setpoint: $30 \%$ of the solar radiation on the building surfaces with no skin system is the threshold after which a responsive feedback mechanism will be activated in order to counteract the deviation (30\% is a design decision by the authors; this number can be modified accordingly in other scenarios).

- Receptor: Building forms have been created algorithmically, allowing for the evaluation of the solar radiation on the building surfaces.

- Effector: If the amount of solar radiation exceeds 30\%, a new morphological attribute, indentation, will be activated on the exposed building surfaces (protrusions). 
Table 3. Homeostatic mechanism B functions.

\begin{tabular}{ll}
\hline Functions and variables & Description \\
\hline $\mathrm{X}$ & The generated phenotype in each iteration \\
$\mathrm{K}()$ & $\begin{array}{l}\text { Receptor. The function that returns the parts of the phenotype with no skin that } \\
\text { receive solar radiation more than } 30 \%\end{array}$ \\
$\mathrm{H}()$ & Effector. The function that applies the morphological changes onto the phenotype \\
\hline
\end{tabular}

$\operatorname{if}(\mathrm{F}(\mathrm{X}) !=$ null $)$

\{

$\operatorname{if}(\mathrm{K}(\mathrm{G}(\mathrm{X})) !=$ null $)$

$\mathrm{H}(\mathrm{G}(\mathrm{X}))$;

else

$\mathrm{G}(\mathrm{X})$;

\}

else

\{

if $(\mathrm{K}(\mathrm{X}) !=$ null $)$

$\mathrm{H}(\mathrm{X})$;

else

$\mathrm{X}$;

\}

\section{Fitness objectives}

The fitness objectives that are driving the generative simulation are as follow (Table 4):

Given the complexity of the design problem, the experiment was limited to 25 individuals per generation with a total number of 300 generations.

\section{Results}

\section{Ablation study}

Five test scenarios with the same algorithmic settings as Table 1 have been conducted to study to what extent the added homeostatic mechanisms will influence the generative simulation. 
Table 4. Fitness functions.

Fitness function

Figure

$\mathrm{FOI}=\mid$ Area $a_{\text {ABahr Towers }}-\sum_{n=0}^{n}$ Area $_{n} \mid$

FOI: To keep the gross floor area (GFA) of the generated solutions as close as possible to the case study.

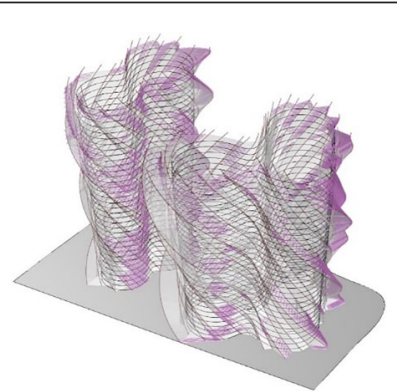

$\delta=\sum_{n=0}^{n} P_{a n} \theta=\sum_{n=0}^{n} P_{s n} F O 2=\left(\frac{\delta}{\theta}\right)$

FO2: To increase the shadow on the buildings by selfshading mechanisms (ratio of all sample points to the blocked sample points).

$\mu=\sum_{n=0}^{n} P G_{a n} \omega=\sum_{n=0}^{n} P G_{s n} F O 3=\left(\frac{\mu}{\omega}\right)$

FO3: To increase the shadow on the ground (ratio of all sample points to the blocked sample points).

$\alpha=\sum_{n=0}^{n} V_{a n} \beta=\sum_{n=0}^{n} V_{p n} F O 4=\left(\frac{\alpha}{\beta}\right)$

FO4: To increase the view from inside of the buildings towards the outside (ratio of all vectors to the blocked vectors). Darker cells illustrate the blocked views.
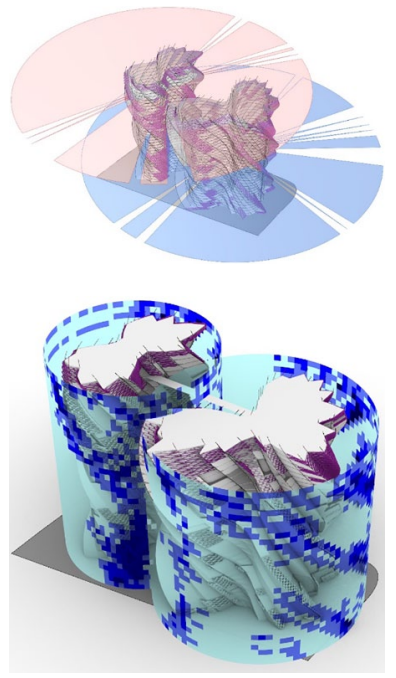
According to the results in Table 5, the performance of the simulation No. 3 is very close to the performance of the simulation No. 1. Simulations No. 2 and No. 4 due to the absence of F () and G (), are missing the skin system on the form, while in simulation No. 5, due to the lack of $F$ () while executing G(), the skin is excessively applied to the entire phenotype. Simulation No. 3 (the bold row) is the simulation with both homeostatic mechanisms activated.

It is evident in Figure 6 that by keeping the Homastasis Mechanism A and B activated during the simulation, the results of the fitness objectives are improved.

\section{The main simulation run}

By running the evolutionary simulation for the main design problem, 7500 genotypes/phenotypes with four fitness values per solution were produced. The Pareto optimal solutions as it is shown as the blue mesh in the objective space in Figure 7, are distributed uniformly throughout the simulation, highlighting the conflict between fitness criteria and an inability for one objective to drive the simulation more than the others.

Figure 8 illustrates four fitness objective performance in four different graphs of Standard deviation graphs, Fitness values graphs, SD value trend line and Mean value trend line. Mean value trendline graphs plot the average fitness value per generation. The mean value for FO2 is decreased and optimised in the generative process. The mean value for FO3 and FO4 from the beginning to the end of the process while having a high frequency of changes did not change drastically however higher SD values for FO2 and FO4 show the variation generated in the solutions set.

\section{Homeostatic mechanism}

To understand to what extent the homeostatic mechanisms were activated and generated the solutions with desired formal attributes and performance, a group of Pareto front solutions of the entire simulation were selected for further investigations and analysis (Figure 9). Each solution is accompanied by a set of analysis and the illustration of their morphological properties. The investigation of each selected solution continues by studying how successfully or unsuccessfully they performed in the following measurement criteria in comparison to the case studies:

- What percentage of the building surface area will receive more than the threshold of $2 \mathrm{KwH} / \mathrm{m} 2$ solar radiation? Due to the chosen date of the analysis (21st June), the sun angle is vertical to the buildings, and the roofs receive most of the solar radiation. According to the annual maximum solar gain in Abu-Dhabi, ${ }^{20} 6.3 \mathrm{KwH} / \mathrm{m} 2$ is the maximum solar gain per day in a year with considering roofs in the calculations. Since in neither case studies nor selected solutions, roofs were in the scope of radiation studies, the number $2 \mathrm{KwH} / \mathrm{m} 2$ was chosen to conduct a meaningful comparison between solutions.

- How much does the evolved skin system obstruct the view from the inside to the outside of the buildings? To conduct a meaningful analysis between the selected solutions and the case studies, the frame of the skin systems in all cases are the only obstructing geometry for calculating the view.

- How well do the selected solutions provide shading on the ground for outdoor activities? This criterion is selected to study how well the selected solutions could enable outdoor activities by shading on the ground in comparison to the case studies. It is an attribute that is missing in the case studies.

In all three measurement criteria, selected candidates perform better than the case studies while exhibiting a wide range of morphological variations. Figure 9 shows the measurement criteria based on which the case 


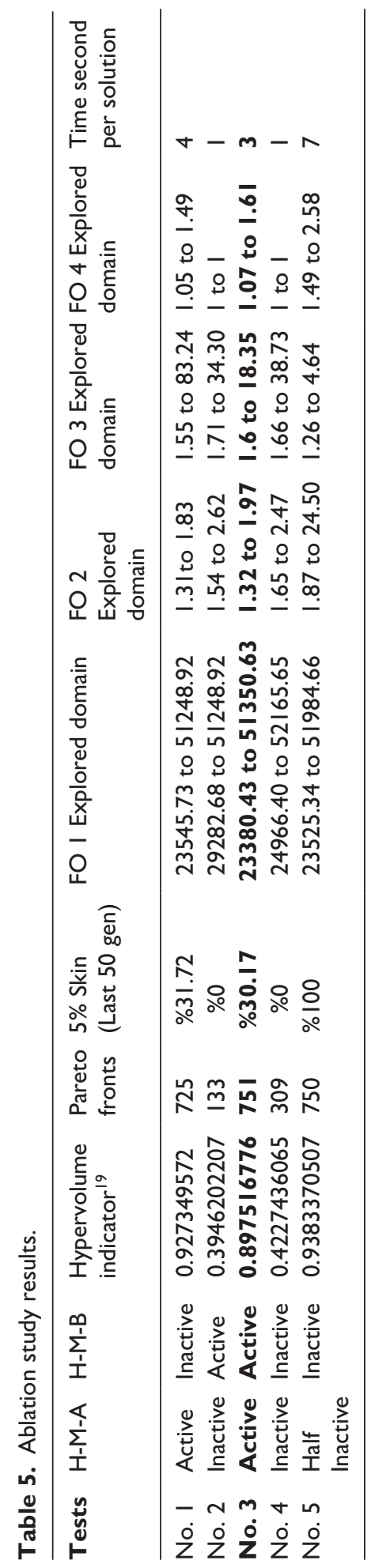




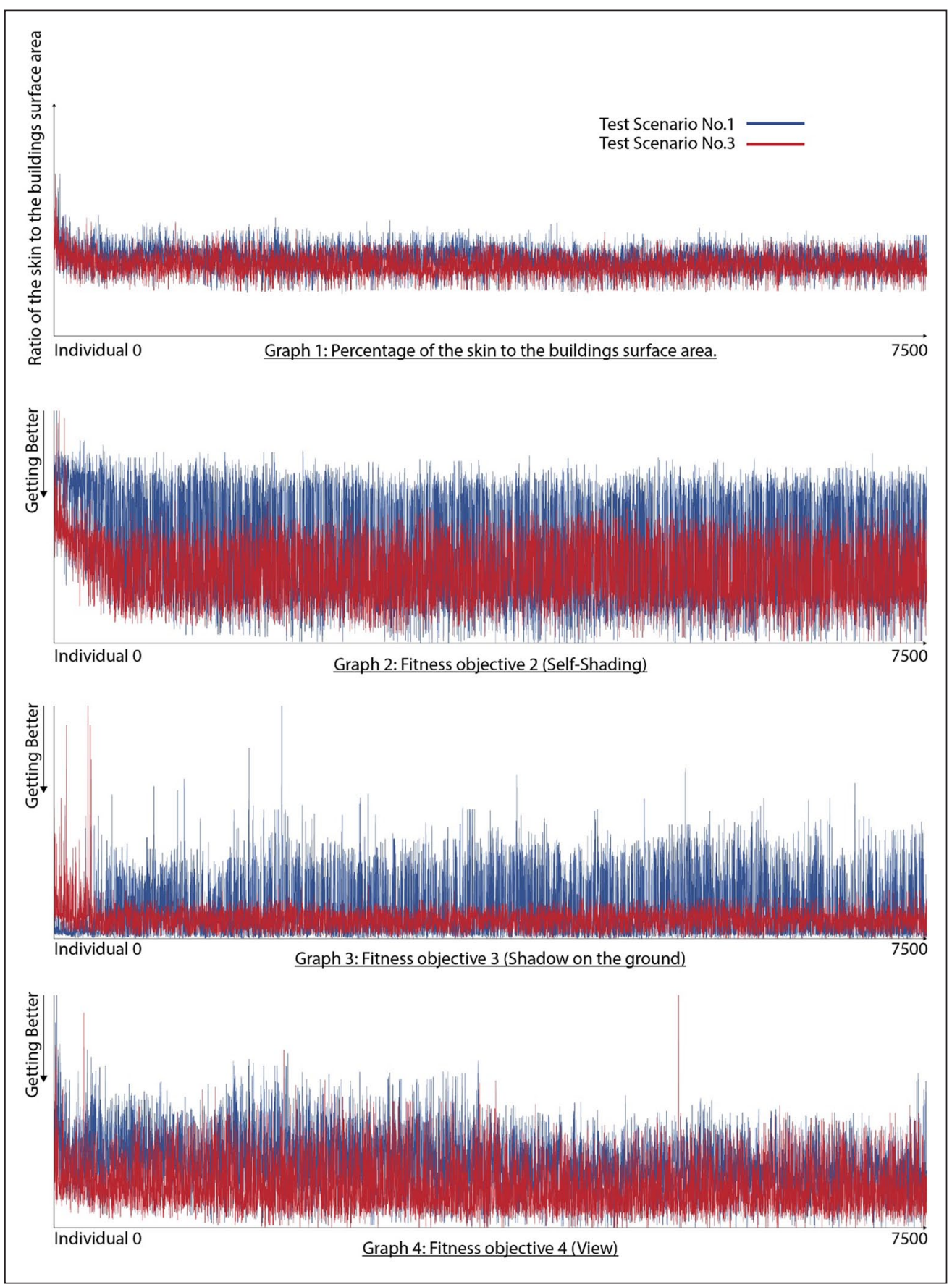

Figure 6. Graph I shows the ratio of the skin to the entire buildings in each iteration. Test scenario 3 generated slightly less skin on the buildings compare to test scenario I. Graphs 2, 3 and 4 show the test scenario 3 is performing better in generating solutions. 


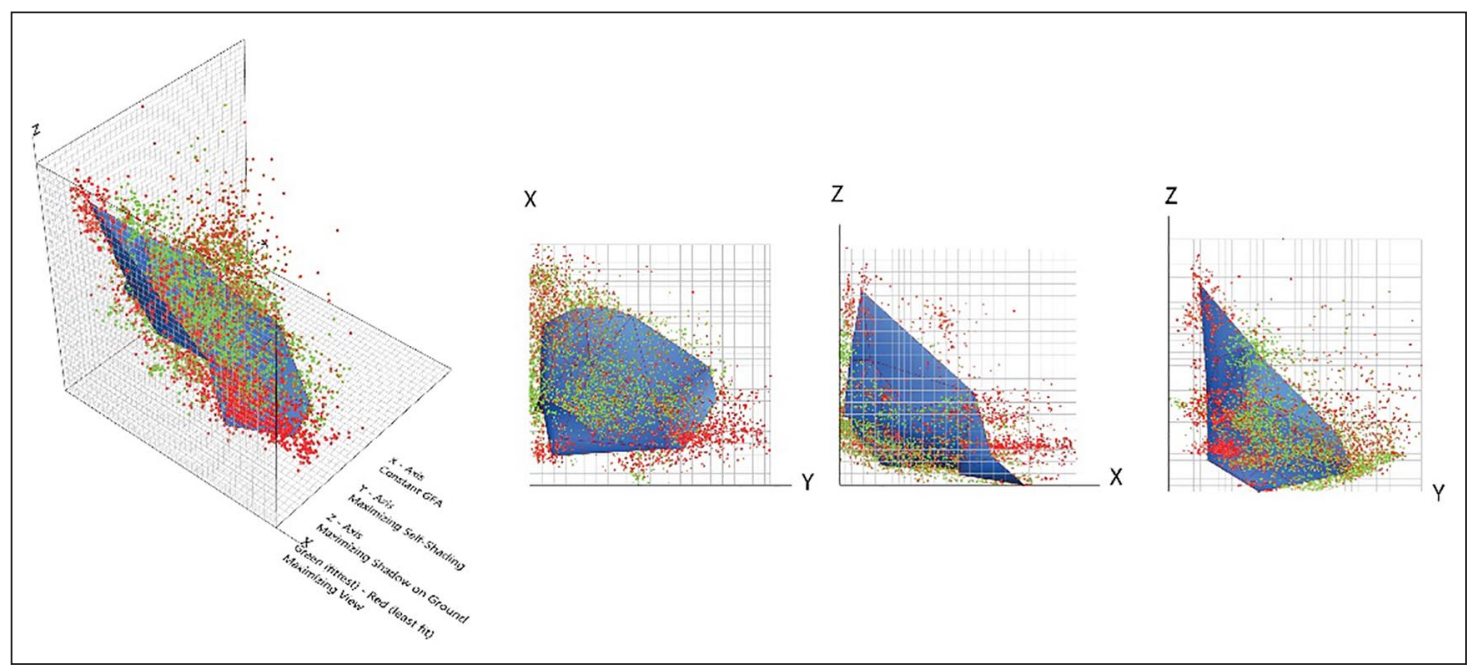

Figure 7. The objective space and the Pareto front mesh.

studies will be compared to the generated solutions in four ways, (1) morphological illustrations, (2) the percentage of the building surface area that receives more than $2 \mathrm{Kwh} / \mathrm{m} 2$ solar radiation, (3) the percentage of the skin that blocks the view from inside to outside and(4) the shadow on the ground (solar radiation analysis were carried out by Ladybug Tools ${ }^{21}$ ).

\section{Discussion}

The research has undertaken the analyses of a generative design system, using principles of regulation and adaptation, to conceive building clusters that are capable of adjusting to high levels of solar radiation. The generative design workflow was modified to facilitate the application of homeostatic principles in its process to generate morphological characteristic in the phenotypes for adaptation to extreme solar radiation.

The case study chosen was The Al-Bahr Towers, because of both the excessive solar radiation which they are subjected to and their use of contemporary systems to regulate temperature. The investigation concluded that the generative design system produced a set of design proposals with morphological characteristics that perform better (based on the measurement criteria) than the case study at a significant level.

Limitations identified throughout the research were primarily related to the long time required for the comparative simulations due to the inclusion of additional conditional statements. There were also some slight errors in the computation which resulted in excessive application or dismissal of skin, leading to 10 per cent of solutions being considered anomalies despite the skin being chosen for its capacity to adapt to complex generated forms.

In order to establish the maximum efficacy of the design mechanism to embed principles of homeostasis, additional research is necessary. Although using feedback loops by including additional conditional statements would add to the required computation time, this addition would allow more solutions to be generated within the simulation in the correct performance range. The authors are currently undertaking more in-depth studies on the improvement of the use of regulatory mechanisms and feedback loops in generative design systems. Moreover, authors are investigating the efficiency of the proposed homeostatic generative design system in different environmental scenarios as well as in design problems with different degrees of complexities. 


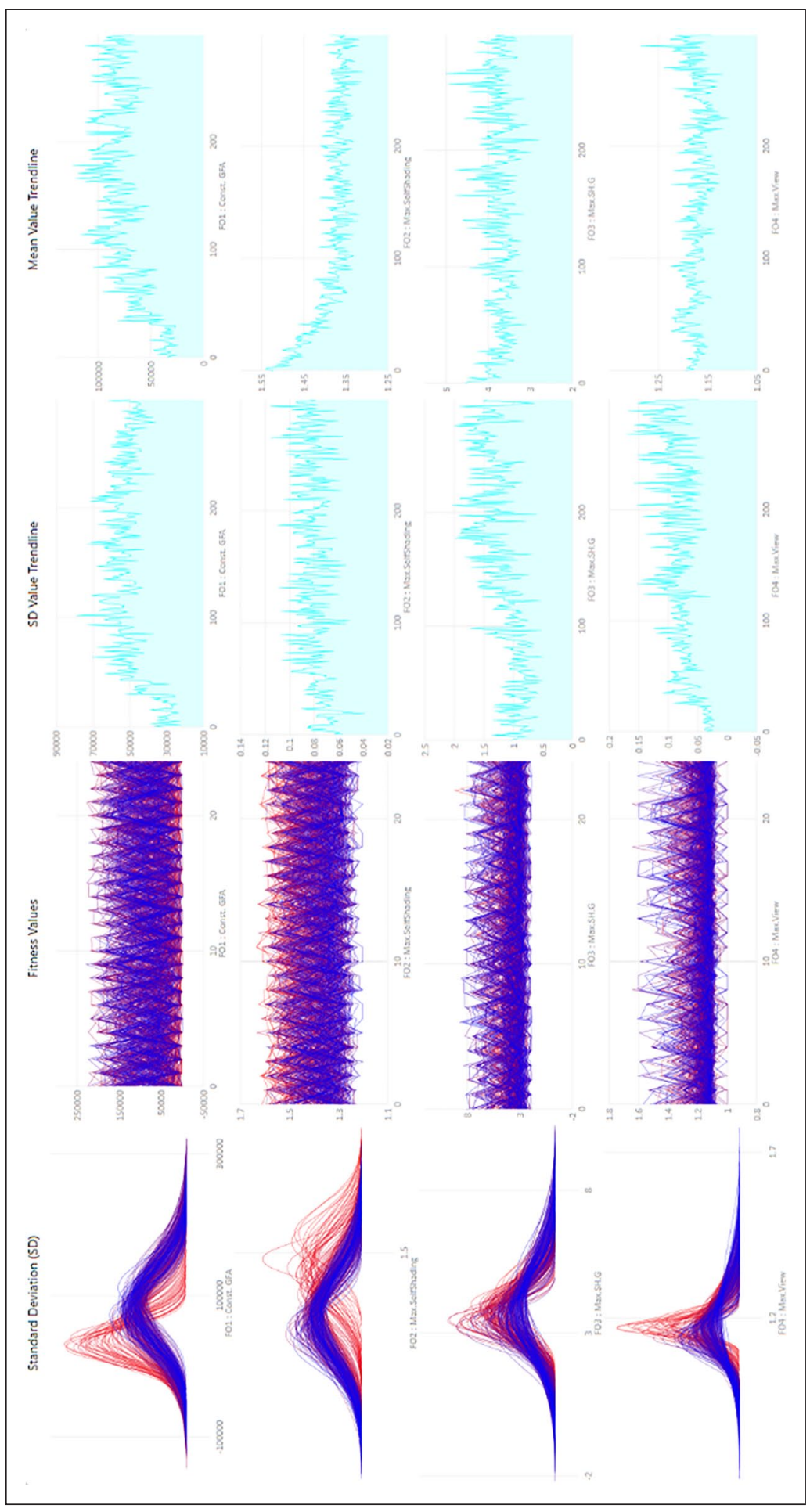

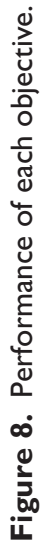




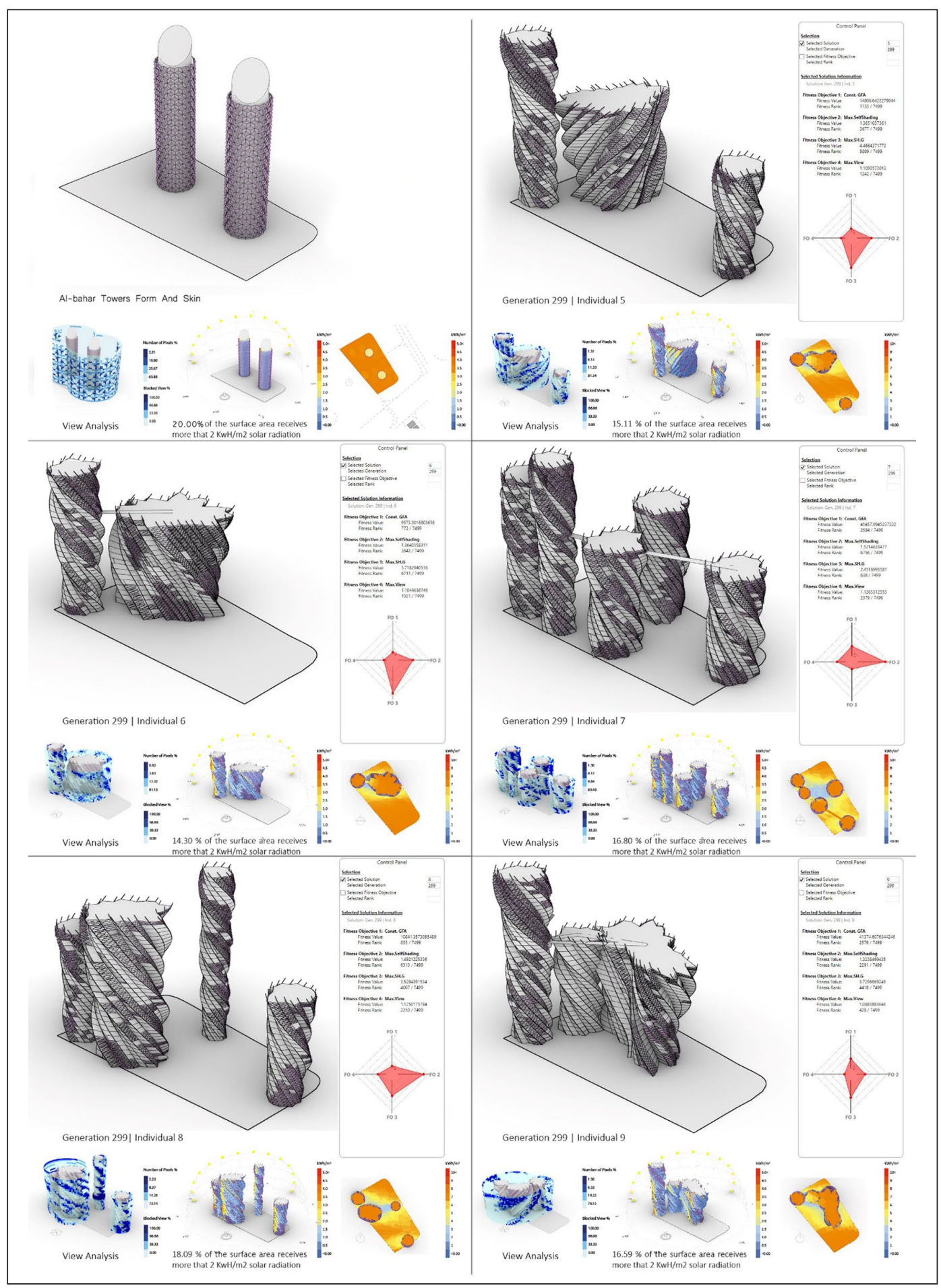

Figure 9. The Al-Bahr towers and the selected number of Pareto fronts and their performance measurements. All of the selected candidates are performing better than the Al-Bahr towers. 


\section{Declaration of conflicting interests}

The author(s) declared no potential conflicts of interest with respect to the research, authorship, and/or publication of this article.

\section{Funding}

The author(s) received no financial support for the research, authorship, and/or publication of this article.

\section{ORCID iD}

Milad Showkatbakhsh (iD https://orcid.org/0000-0001-5440-5175

\section{References}

1. European Commission (ed). Energy roadmap 2050. Luxembourg: Publications Office of the European Union, 2012, p. 20.

2. Napier J. Climate based façade design for business buildings with examples from central London. Buildings 2015; 5(1): 16-38.

3. Attia S. Evaluation of adaptive facades: the case study of Al Bahr towers in the UAE. QScience Connect 2018; 2017(2): 6.

4. Horizon 2020 [Internet]. Horizon 2020 - European Commission, https://ec.europa.eu/programmes/horizon2020/en (2020, accesssed 8 June 2019).

5. B Cannon W. Wisdom of the body. Rev. and Enl. Ed edition. New York: W. W. Norton and Company, Inc., 1963, p. 340.

6. Gerlee P, Basanta D and Anderson ARA. Evolving homeostatic tissue using genetic algorithms. Progr Biophys Mol Biol 2011; 106(2): 414-425.

7. Rye C, Wise RR, Jurukovski V, et al. Biology. Houston, TX: Rice University, 2017.

8. Steadman P. The evolution of designs: biological analogy in architecture and the applied arts. London and New York: Routledge, 2008, p. 485.

9. Torday J. Homeostasis as the mechanism of evolution. Biology 2015; 4(3): 573-590.

10. Wright S. The roles of mutation, inbreeding, crossbreeding, and selection in evolution. In: 6th International Congress on Genetics. New York: Brooklyn botanic garden, 1932, pp. 356-366.

11. Mayr E. Toward a new philosophy of biology: observations of an evolutionist. Cambridge, MA: Harvard University Press, 1988.

12. Deb K, Agrawal S, Pratap A, et al. A fast elitist non-dominated sorting genetic algorithm for multi-objective optimisation: NSGA-II. In: International conference on parallel problem solving from nature. Paris, France: Springer, 2000, pp. 849-858.

13. Makki M, Showkatbakhsh M and Song Y. Wallacei [Internet], https://www.wallacei.com (2019, accessed 27 May 2019).

14. Makki M, Showkatbakhsh M, Tabony A, et al. Evolutionary algorithms for generating urban morphology: variations and multiple objectives. Int J Archit Comput 2018; 17(1): 1-31.

15. Makki M, Showkatbakhsh M and Song Y. Wallacei primer 1.0, www.wallacei.com (2019).

16. Pal JS and Eltahir EAB. Future temperature in southwest Asia projected to exceed a threshold for human adaptability. Nat Climate Change 2016; 6(2): 197-200.

17. Al Bahr Towers, Abu Dhabi, UAE - KonceptX [Internet], http://konceptx.com/portfolio/al-bahr-towers-abu-dhabiuae/ (accessed 2 June 2020).

18. Karanouh A and Kerber E. Innovations in dynamic architecture. J Facade Design Eng 2015; 3(2): 185-221.

19. Bader J and Zitzler E. HypE: An algorithm for fast hypervolume-based many-objective optimisation. Evol Comput 2011; 19(1): 45-76.

20. Bankable solar data for better decisions, https://solargis.com/ (accessed 23 September 2019).

21. Sadeghipour Roudsari M and Mackey C. Ladybug tools | Home Page [Internet], https://www.ladybug.tools/ (accessed 11 June 2019). 